\title{
Exploring Memory Interventions in Depression through Lifelogging Lens
}

\author{
Chengcheng Qu \\ Lancaster University \\ Lancaster, UK \\ c.qu3@lancaster.ac.uk
}

\author{
Corina Sas \\ Lancaster University \\ Lancaster, UK \\ c.sas@lancaster.ac.uk
}

\begin{abstract}
Depression is a major affective disorder with significant socio-economic cost. Distinctive autobiographical memory impairments in depression include overgeneralization, negative-bias, and repetitive negative thinking. A few psychotherapeutic interventions have been purposefully designed to address these impairments, albeit they benefit from limited technological support. This paper reports an analysis of four memory-based interventions proven effective in the therapeutic practice targeting depression. We also explored the memory impairments addressed by these interventions. Our findings led to three design implications for digital tools in this space. We suggest the value of supporting enriched positive memory recall, negative memory reappraisal, and future episodic imagination.
\end{abstract}

Memory impairments, depression, overgeneralization, negative bias, rumination, lifelogging technologies

\section{INTRODUCTION}

With significant social and economic costs, depression is a major affective disorder impacting over 300 million people worldwide (WHO, 2012). Depression influences cognitive functions leading to distinctive memories impairments (Köhler et al., 2015). People living with depression have overgeneralized autobiographical memories with a strong bias towards negative events (Dalgleish et al., 2014). Several interventions have proven effective in addressing such memory deficits in depression (Table 1). These consist of Memory Specificity Training (MEST) (Raes et al., 2009), Concreteness Training (CNT) (Watkins et al., 2012), Competitive Memory Training (COMET) (Korrelboom et al., 2012) and Imagery Cognitive Bias Modification (CBM-I) (Holmes et al., 2013).

Most $\mathrm{HCl}$ research on depression has focused on developing computerised cognitive interventions, such as online Cognitive Behavioural Therapy (CBT) (Coyle et al., 2007 and Doherty et al., 2012). Limited work, however, has explored how memory technologies could support memory-related cognitive challenges in mood regulation, especially for preventing and alleviating depression. This is surprising given that lifelogging technologies could capture and effectively support autobiographical memory retrieval through records of pictures, audio, or affective states of memories (Van den Hoven et al., 2008 and Sellen et al., 2010). This paper explores four psychotherapeutic interventions for memory impairments in depression and the feasibility of augmenting them with digital tools (Table 2).

\section{RELATED WORK}

\subsection{Mental Health in $\mathrm{HCl}$}

Much $\mathrm{HCl}$ research has explored prevention, detection and treatment of mood disorders, with a focus on emotional and behavioural responses. For example, social media has been used to detect depression (Tsugawa et al., 2015), while digital affective diaries (Konrad et al., 2016) and expressive writing method (Pennebaker et al., 2017) have been developed to support monitoring and reflecting on one's experience. Technologies have usually been intended as aids for traditional therapeutic

Table 1. Four interventions targeting memory impairments in depression

\begin{tabular}{|l|l|l|}
\hline \multicolumn{1}{|c|}{ Intervention } & \multicolumn{1}{|c|}{ Main procedure } & $\begin{array}{l}\text { Addressed memory } \\
\text { deficits }\end{array}$ \\
\hline $\begin{array}{l}\text { Memory Specific } \\
\text { Training (MEST) }\end{array}$ & $\begin{array}{l}\text { Guided by trained therapists, this intervention presents a series of cue } \\
\text { words, and asks people to retrieve as many specific memories as possible }\end{array}$ & Overgeneralization \\
\hline $\begin{array}{l}\text { Concreteness Training } \\
\text { (CNT) }\end{array}$ & $\begin{array}{l}\text { This intervention requires people to repeatedly imagine the sensory details, } \\
\text { warning signs, and actions related to difficult events and to reflect on them } \\
\text { in a concrete manner. }\end{array}$ & $\begin{array}{l}\text { Overgeneralization, } \\
\text { rumination }\end{array}$ \\
\hline $\begin{array}{l}\text { Competitive memory } \\
\text { training (COMET) }\end{array}$ & $\begin{array}{l}\text { This intervention asks people to identify a positive self-identity, enrich it } \\
\text { iteratively and practice such imagination over several sessions. }\end{array}$ & $\begin{array}{l}\text { Rumination, negative } \\
\text { bias (negative self- } \\
\text { image) }\end{array}$ \\
\hline $\begin{array}{l}\text { Imagery Cognitive Bias } \\
\text { Modification (CBM-I) }\end{array}$ & $\begin{array}{l}\text { This intervention asks people to imagine themselves involved in prescribed } \\
\text { scenes. The scenes start ambiguously and are resolved positively. }\end{array}$ & $\begin{array}{l}\text { Negative bias (future- } \\
\text { oriented scenario) }\end{array}$ \\
\hline
\end{tabular}


interventions, harnessing the potential of augmented reality (Wrzesien et al., 2015), interactive games (Coyle et al., 2011), or multimedia positive selfportraits (Mynatt et al., 2001). More specifically regarding depression, $\mathrm{HCl}$ work has focused on online CBT (Coyle et al., 2007 and Doherty et al., 2012) for delivering digital training content. Limitations of these approaches are twofold. First, they have focused mostly on the affective and behavioural aspects of mental health and less on its cognitive aspects. Second, the few studies exploring cognition-based interventions in depression, such as online CBT, have employed technologies with limited interactivity, personalisation, or user engagement (Doherty et al., 2012).

\subsection{Memory Technologies}

Much $\mathrm{HCl}$ work has explored the value of lifelogging technologies in memory augmentation, by supporting memory capture and retrieval. Such technologies harnessed wearable sensors including, for example, SenseCam (Hodges et al., 2011), narrative clip (Viet Le et al., 2016) or biosensors (Sas et al., 2013) to cue retrieval of autobiographical memories. Such exhaustive and multimodal capture underpins the effectiveness of lifelogging technologies in addressing memory impairments (Sas et al., 2015). Lifelogging tools have also been explored in relation to progressive neurological disorders, which lead to significant memory impairments, such as Alzheimer's disease and dementia (Harvey et al., 2015, Allé et al., 2017 and L. Lee and K. Dey, 2008).

However, unlike other conditions involving memory challenges, memories of people living with depression are not necessarily impaired but rather difficult to access from the autobiographical memory structure (Conway, 2005). As Conway identified in his autobiographical memory model, the accessibility of specific episodic memories depends on one's working self, which is a complex set of self-images and active goals. Difficulty in memory retrieval is an important marker of depression (Dalgleish et al., 2014). Such difficulties consist of overgeneralized memories leading to biased interpretation and imagination of future events, as well as negativebiased memory retrieval. More specifically, people with depression have unique difficulties in retrieving specific episodic memories. They have a high tendency to truncate their memory-searching process and only recall overgeneralized memory in a negative tone, with a low level of specificity (Williams et al., 2007). Another distinctive memory impairment in depression is that negative memories are faster and richer to retrieve, while positive ones are slow and difficult to retrieve, less vivid and low in emotional response (Dalgleish et al., 2014). Thus, people living with depression may benefit little from simply reviewing sensory details of their memories prompted by lifelogging-based cues. They require a rather complex process to enhance their abilities in recalling and reflecting on their memories, to reexperience positive emotions associated with their happy memories and disengage with the negative emotions from their sad memories.

\section{METHOD}

Our study consists of an analysis of the four identified memory-based interventions proven effective in alleviating depression in lab settings. The selection of the four interventions: CNT (Watkins et al. 2012), MEST (Raes et al., 2009), COMET (Korrelboom et al., 2012), and CBM-I (Holmes et al., 2013) is based on the findings of a recent metaanalysis of autobiographical memory based interventions for depression (Hitchcock et al., 2017). Our analysis aims to reveal the main cognitive challenges addressed by these interventions, in terms of the cognitive abilities targeted by each intervention, especially for coping with emotional events and memories. The aim is to explore if and how lifelogging tools could better support these interventions.

Table 2. Four interventions and their potential of integrating with lifelogging technologies.

\begin{tabular}{|c|c|c|c|c|c|}
\hline \multirow{2}{*}{$\begin{array}{l}\text { Interventi } \\
\text { on }\end{array}$} & \multirow{2}{*}{$\begin{array}{l}\text { The key value to } \\
\text { memory abilities }\end{array}$} & \multicolumn{2}{|r|}{ Input } & \multicolumn{2}{|r|}{ Output } \\
\hline & & Current & Lifelogging-augmented & Current & Lifelogging- augmented \\
\hline \multirow[t]{2}{*}{ MEST } & \multirow{2}{*}{$\begin{array}{l}\text { Enhancing specific } \\
\text { memory recollection }\end{array}$} & \multirow{2}{*}{$\begin{array}{l}\text { Emotional cue } \\
\text { words }\end{array}$} & Bank of emotional memory cues & \multirow{2}{*}{$\begin{array}{l}\text { Verbal } \\
\text { discussion }\end{array}$} & \multirow{2}{*}{$\begin{array}{l}\text { Visualize a bank of } \\
\text { recollected specific } \\
\text { emotional memories. }\end{array}$} \\
\hline & & & Personalized emotional cues & & \\
\hline \multirow[t]{2}{*}{ CNT } & \multirow{2}{*}{$\begin{array}{l}\text { Reviewing } \\
\text { memories and } \\
\text { change viewpoint }\end{array}$} & \multirow{2}{*}{$\begin{array}{l}\text { Audio } \\
\text { training\& first- } \\
\text { aid relaxation } \\
\text { guide }\end{array}$} & $\begin{array}{l}\text { Bank of negative memories to } \\
\text { be analysed }\end{array}$ & \multirow[t]{2}{*}{$N / A$} & \multirow{2}{*}{$\begin{array}{l}\text { Identify user's negative } \\
\text { thinking pattern. Detect } \\
\text { and offset rumination } \\
\text { from an early stage }\end{array}$} \\
\hline & & & $\begin{array}{l}\text { An interactive guide for } \\
\text { reflection on negative memories }\end{array}$ & & \\
\hline COMET & $\begin{array}{l}\text { Enhance positive } \\
\text { memories } \\
\text { recollection, build a } \\
\text { positive self-image }\end{array}$ & $\begin{array}{l}\text { Self- } \\
\text { generated } \\
\text { materials }\end{array}$ & $\begin{array}{l}\text { Bank of positive memories } \\
\text { across the lifespan }\end{array}$ & $\begin{array}{l}\text { Imagery } \\
\text { positive } \\
\text { self-image }\end{array}$ & $\begin{array}{l}\text { Visualize an ideal self- } \\
\text { image with enriched } \\
\text { sensorial details. }\end{array}$ \\
\hline CBM-I & $\begin{array}{l}\text { Enhance positive } \\
\text { current/ future } \\
\text { imagination with } \\
\text { everyday scenes. }\end{array}$ & $\begin{array}{l}\text { Neutral scene } \\
\text { via audio or } \\
\text { pictorial } \\
\text { materials }\end{array}$ & $\begin{array}{l}\text { Personalized ambiguous scenes } \\
\text { from "editing" users' everyday } \\
\text { neutral scene }\end{array}$ & $N / A$ & $\begin{array}{l}\text { Visualize users' } \\
\text { imagination as training } \\
\text { result (e.g. painting). }\end{array}$ \\
\hline
\end{tabular}




\section{FINDINGS}

\subsection{The Challenge of Recollecting Specific Episodic Memories}

MEST and CNT both pointed out that people living with depression suffer from categorical memory recollection in a negative tone. The overgeneralization process stops individuals from reflecting on what had happened, but instead keep processing abstract negative memories. Overgeneralization, as an indicator of depression, could persist even after remission from depression and potentially contribute to the next depressive episode. As an intervention specifically targeting this cognitive vulnerability, MEST trains participants' ability to retrieve specific episodic memories in detail. It provides word-based cues associated with different emotional valence, to break people's categorical negative thinking. CNT trains participants' abilities for reviewing specific negative memories and for re-evaluating them to mitigate against overgeneralization.

\subsection{The Challenges of Building a Positive Self- image}

People living with depression tend to have negative views of their lives, keep revisiting their negative life events, and feel like only bad things happened in their life. The negative-biased view contributes to their negative self-image, as pointed out and addressed by COMET. According to Conway's autobiographical memory theory (Conway, 2005), people evaluate and interpret their present and future scenarios by retrieving relevant memories. Therefore, as addressed by CBM-I, people who hold a negative view of their lives tend to interpret events in a consistently negative way, which contributes to their low motivation and hopelessness. During COMET intervention, participants first identify negative self-identity and imagine an incompatible positive self-identity. They then strengthen this positive self-image by progressively adding verbalisation, body postures, background music, and facial expressions. CBM-I exposes participants to neutral scenes, presented acoustically and visually, and asks participants to imagine a vivid positive ending to the described neutral scenes. This intervention also engages people in positive imagination.

\subsection{The Challenge of Disengaging from Negative Thoughts}

Due to cognitive weaknesses such as negative bias and overgeneralization, people living with depression have difficulties in disengaging from repetitive negative thoughts. CNT supports people to review and reflect on specific difficult events and analyse the triggers of negative emotions. This intervention also encourages participants to explore alternative solutions and to alter the viewpoints of their upsetting memories. COMET trains participants' ability for retrieving positive memories as alternative material against negative thoughts. The increased accessibility of positive memories could reduce the possibility for negative memories to hijack one's cognitive resources.

To summarise (Figure 1), these findings indicated that memory-based interventions could benefit from lifelogging technologies. Key here is to identify the right cue content, which should (i) address negative bias (Dalgleish et al., 2014), (ii) address the identified challenges of the above interventions, and (iii) leverage the potential of lifelogging tools to enable personalized, self-tailored and more engaging interventions (Doherty et al., 2012).

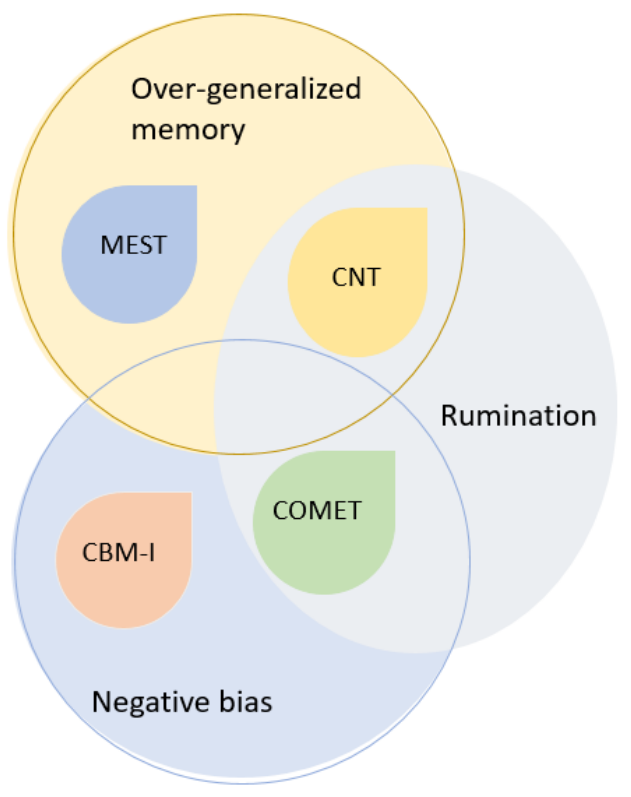

Figure 1. This figure illustrates target symptoms of each intervention: MEST and CNT target overgeneralization. COMET and CBM-I aim to reduce negative bias, while CNT and COMET aim to reduce rumination.

\section{DESIGN IMPLICATIONS}

\subsection{Supporting Sensory Rich Positive Recall (Challenge 1, 2)}

As pointed out by MEST and COMET, people living with depression face difficulties both in retrieving positive memories and in reliving associated positive emotions. They tend to have insufficient positive memories to build a confident self-concept against their negative one. Therefore, one can think of new tools for capturing and archiving positive events across the lifespan. Such a multimodal bank of positive memories, captured through biosensors could be deliberately tagged with sensorial contents, for example, audio, visual, or haptic, close to the 
moment of encoding. Such a positive memory bank has the potential to contribute to vivid positive selfimagination. It can also strengthen people's memory processing abilities, especially the encoding of positive episodic memories. This in turn can safeguard people from the offset of depression.

Unlike generic cues used in MEST intervention, this positive memory bank, with enriched personal memories could provide powerful self-relevant cues (Sas, 2018) simulating the memory-encoding context, which also harnesses the contextdependent quality of memory for better recall when the contexts of encoding and retrieval are similar (Tulving, 1973). Personalized training modules, as pointed by previous online mental health intervention research (Doherty et al., 2012) is also important for user engagement. In addition, a bank of positive memories could support the visualisation of users' training progress and provide them with a sense of achievement.

\subsection{Supporting Reflection on Negative Memories (Challenge 3)}

The above interventions point out a design space for supporting reflection on mood journal. As previous $\mathrm{HCl}$ studies about mood journals revealed (Konrad et al., 2016 and Hollis et al., 2017), simply reviewing previously written memories is not enough for mood regulation. Designers of such systems also need to consider the mood dependent context and human memory weakness (Sellen and Whittaker, 2010). For example, findings from mood journal studies (Konrad et al., 2016) have shown that reflecting on positive memories in sad moods could potentially taint the happy memories and induce joy-killing thoughts. Findings also indicated that revisiting negative memories from digital affective diaries (Hollis et al., 2017) contributes little to both momentary and long-term mood improvement, although it has proven effective in pencil and paper based studies (Pennebaker et al., 2017) for inducing re-appraisal, self-distancing and wellbeing.

Thus, it would be useful to support users to reflect on their memories in a more structured manner by replacing their previous free-style of reflection. For example, CNT inspires a design opportunity for a novel interactive system that explicitly facilitates reflection and sense-making, by integrating natural language processing techniques such as conversational agents for guiding such reflection in a concrete style. If used along the lifespan, the system may allow early identification and correction of negative thinking patterns. The system is promising for detecting negative thinking patterns at early stages of rumination. Such a system could thus offsetting the negative influence of recalling traumatic events, and potentially preventing the depressive episode to set off.

\subsection{Supporting Positive Reinterpretation and Imagination (Challenge 2)}

Our findings indicate the value of CBM-I training in supporting the positive interpretation of generic ambiguous events. As suggested in $\mathrm{HCl}$ mental health research (Doherty et al., 2012), personalisation is key to user engagement. We can thus think of exploiting lifelogging technologies for collecting self-relevant everyday neutral events or "editing" anticipated ambiguous future events to train positive interpretation. For example, when users want to reflect on an emotional event, the system could remove the colour of a captured pictorial cue and ask users to i) colour the picture themselves, and then ii) draw an imaginary positive ending of this ambiguous scene. This process, inspired by mandala colouring as art-therapy (Palmer et al., 2014) could lead users to an engaging future-oriented positive imagination practice. Authoring tools can also be envisaged to assist people in capturing their interpretation of such events over their entire lifespan. Users could thus pay more attention to their everyday scenes and be more engaged in the present (Keyes, 2014).

We argue that technologies such as lifelogging are well suited to address the negative bias in depression. However, more effort should be put in leveraging potential of such technologies to address overgeneralized memories, especially identifying positive memories as material to guard against negative overgeneralization. In addition, identifying neutral events to train positive interpretation could also have great potential in addressing memory impairments in depression.

\section{CONCLUSION}

This work explores the potential of lifelogging technologies to support memory-based interventions in depression. We report an analysis of four such interventions. Findings identified the cognitive challenges targeted by each of them, as well as their training procedures, materials and main limitations. We employed a "lifelogging lens" to this analysis and identified three design implications addressing the challenge of negative bias of memory retrieval and interpretation in depression.

\section{ACKNOWLEDGEMENT}

This work has been supported by AffecTech: Personal Technologies for Affective Health, Innovative Training Network funded by the H2020 Marie Skłodowska-Curie GA No 722022. 


\section{REFERENCES}

Mélissa C. Allé, Liliann Manning, Jevita Potheegadoo, Romain Coutelle, Jean Marie Danion, and Fabrice Berna. 2017. Wearable Cameras Are Useful Tools to Investigate and Remediate Autobiographical Memory Impairment: A Systematic PRISMA Review. Neuropsychology Review 27, volume 27, issue 1, page 81-99.

Martin A Conway. 2005. Memory and Language Memory and the self. Memory and the self. Journal of Memory and Language, volume 53, issue 4, page 594-628.

Corey L. M. Keyes. 2014. Mental Health as a Complete State: How the Salutogenic Perspective Completes the Picture. Bridging Occupational, Organizational and Public Health, page 179-192.

David Coyle, Gavin Doherty, Mark Matthews, and John Sharry. 2007. Computers in talk-based mental health interventions. Interacting with Computers, Volume 19, issue 4, page 545-562.

Tim Dalgleish and Aliza Werner-Seidler. 2014. Disruptions in autobiographical memory processing in depression and the emergence of memory therapeutics. Trends in Cognitive Sciences, volumn 18, issue 11, page 596-604.

Gavin Doherty, David Coyle, and John Sharry. 2012. Engagement with online mental health interventions. Proceedings of the 2012 ACM annual conference on Human Factors in Computing Systems - CHI '12, New York, May 2012, page 1421-1430. ACM, New York, NY, USA.

Morgan Harvey, Marc Langheinrich, and Geoff Ward. 2015. Remembering through lifelogging: A survey of human memory augmentation. Pervasive and Mobile Computing, volume 27, page 14-26.

Caitlin Hitchcock, Aliza Werner-Seidler, Simon E. Blackwell, and Tim Dalgleish. 2017. Autobiographical episodic memory-based training for the treatment of mood, anxiety and stress-related disorders: A systematic review and meta-analysis. Clinical Psychology Review, volume 52, page 92-107.

Victoria Hollis, Artie Konrad, Aaron Springer, Matthew Antoun, Christopher Antoun, Rob Martin, Steve Whittaker. 2017. What Does All This Data Mean for My Future Mood? Actionable Analytics and Targeted Reflection for Emotional Well-Being. Human-Computer Interaction, volume 32, issue 5-6, page 208-267.

Cristiano A.Köhler, André F. Carvalho, Gilberto S. Alves, Roger S. Mclntyre, Thomas N. Hyphantis,
Martín Cammarota. 2015. Autobiographical Memory Disturbances in Depression: A Novel Therapeutic Target? Neural Plasticity, volumn 2015, page 1-14.

Artie Konrad, Simon Tucker, John Crane, and Steve Whittaker. 2016. Technology and Reflection: Mood and Memory Mechanisms for Well-Being. Psychology of Well-Being, volume 6 , issue 1 , page 5 .

Kees Korrelboom, Maaike Maarsingh, and Irma Huijbrechts. 2012. Competitive memory training (COMET) for treating low self-esteem in patients with depressive disorders: A randomized clinical trial. Depression and Anxiety, volume 29, issue 2, page 102-110.

Huy Viet Le, Sarah Clinch, Corina Sas, Tilman Dingler, Niels Henze, and Nigel Davies. 2016. Impact of Video Summary Viewing on Episodic Memory Recall - Design Guidelines for Video Summarizations. In Proceedings of the $2016 \mathrm{CHI}$ Conference on Human Factors in Computing Systems - CHI '16. San Jose, California, USA, May 2016, page 4793-4805. ACM, New York, NY, USA.

Matthew L. Lee and Anind K. Dey. 2008. Lifelogging memory appliance for people with episodic memory impairment. Proceedings of the 10th international conference on Ubiquitous computing -UbiComp '08, Seoul Korea, September 2008, page 44-53. ACM New York, NY, USA.

Elizabeth D. Mynatt, Jim Rowan, Sarah Craighill, and Annie Jacobs. 2001. Digital family portraits: supporting peace of mind for extended family members. In Proceedings of the SIGCHI Conference on Human Factors in Computing Systems - CHI '01, Seattle Washington USA, March 2001, page 333-340. ACM, New York, NY, USA.

Victoria J. Palmer, Christopher Dowrick, and Jane M. Gunn. 2014. Mandalas as a visual research method for understanding primary care for depression. International Journal of Social Research Methodology, volume 17, issue 5, page 527-541.

James W. Pennebaker. 2017. Expressive Writing in Psychological Science. Perspectives on Psychological Science, volumn 13, issue 2, page 226-229.

Filip Raes, J. Mark G. Williams, and Dirk Hermans. 2009. Reducing cognitive vulnerability to depression: A preliminary investigation of MEmory Specificity Training (MEST) in inpatients with depressive symptomatology. Journal of Behavior Therapy and Experimental Psychiatry, volume 40, issue 1, page 24-38. 
Corina Sas. 2018. Exploring Self-Defining Memories in Old Age and their Digital Cues. Proceedings on Designing Interactive Systems - DIS'18 (in press).

Corina Sas and Christopher Clarke. 2015. SelfDefining Memory Cues: Creative Expression and Emotional Meaning. Proceedings of the 33rd Annual ACM Conference Extended Abstracts on Human Factors in Computing Systems- CHI EA '15, Seoul Republic of Korea, April 2015, page 2013-2018. ACM, New York, NY, USA.

Corina Sas and Matthew Rees. 2013. AffectCam: Arousal - Augmented SenseCam for Richer Recall of Episodic Memories. Proceedings of the 31rd Annual ACM Conference Extended Abstracts on Human Factors in Computing Systems- CHI EA '13, Paris France, April 2013, page 1041-1046. ACM, New York, NY, USA.

Abigail J. Sellen, Andrew Fogg, Mike Aitken, Steve Hodges, Carsten Rother, and Ken Wood. 2007. Do life-logging technologies support memory for the past? Proceedings of the SIGCHI conference on Human factors in computing systems - CHI '07, San Jose California USA, April 2007, page 81. ACM, New York, NY, USA.

Abigail J. Sellen and Steve Whittaker. 2010. Beyond total capture: A Constructive Critique of Lifelogging. Communications of the ACM, volume 53 , issue 5 , page 70-77.

Tamara J. Lang, Simon E. Blackwell, Catherine J. Harmer, Phil Davison, Emily A. Holmes. 2013. Cognitive Bias Modification Using Mental Imagery for Depression: Developing a Novel Computerized Intervention to Change Negative Thinking Styles. Personality and Information Processing, volume 26, issue 2, page 87-163.

Edward R. Watkins, Rod Taylor, et al. 2012. Guided self-help concreteness training as an intervention for major depression in primary care: a Phase II randomized controlled trial. Psychological Medicine, volume 42, issue 07, page 1359-1371.

J. Mark G. Williams, Thorsten Barnhofer, Catherine Crane, et al. 2007. Autobiographical memory specificity and emotional disorder. Psychological Bulletin, volume 133, issue 1, page 122-148.

Tulving, E., \& Thomson, D. M. (1973). Encoding specificity and retrieval processes in episodic memory. Psychological review, volume 80 , issue 5, page 352-373.

WorldHealth Organization, WHO, 2012. Depression (Fact Sheet 369).World Health Organization, Media Centre. Http://www.who.int/ mediacentre/factsheets/fs369/en/ (Retrieved January 13, 2015).

Steve Hodges, Emma Berry, Ken Woodberry. SenseCam: A wearable camera that stimulates and rehabilitates autobiographical memory. Memory, volume 19, issue 7, 685-696. 